\title{
QUANTITATIVE BEHAVIOUR OF THE NORMS OF AN ANALYTIC MEASURE
}

\author{
LOUIS PIGNO AND BRENT SMITH
}

\begin{abstract}
A Littlewood-Paley type inequality for the quotient norms of an analytic measure is obtained; one consequence of this inequality is the classical theorem of F. and M. Riesz.
\end{abstract}

In this paper $\mathbf{T}$ is the circle group, $\mathbf{Z}$ the additive group of integers and $M(\mathbf{T})$ the customary space of Borel measures on $\mathbf{T}$; for $\mu \in M(\mathbf{T})$ and $n \in \mathbf{Z}$ define

$$
\hat{\mu}(n)=\int_{\mathbf{T}} e^{-i n \theta} d \mu(\theta) .
$$

If $\mu \in M(\mathbf{T})$, put $\mu=\mu_{a}+\mu_{s}$ where $\mu_{a}$ is absolutely continuous with respect to Lebesgue measure on $\mathrm{T}$ and $\mu_{s}$ is singular with respect to Lebesgue measure on $\mathbf{T}$; let $M_{a}(\mathbf{T})$ denote the space of all absolutely continuous measures. A measure $\mu \in M(\mathbf{T})$ is said to be of analytic type if $\hat{\mu}(n)=0$ for all $n<0$; as usual $H^{1}(\mathbf{T})$ denotes the space of all analytic measures on $\mathbf{T}$.

F. and M. Riesz [7] have proved the following result for measures of analytic type.

THEOREM R. If $\mu \in H^{1}(\mathbf{T})$ then $\mu \in M_{a}(\mathbf{T})$.

The purpose of this paper is to present a quantitative version of the $F$. and $M$. Riesz Theorem; before stating our result, we will need two quantitatively explicit lemmas concerning the behaviour of the quotient norms of a measure.

For any $\omega \in M(\mathbf{T})$ and $E \subset \mathbf{Z}$ put

$$
\|\omega\|_{E}=\inf \{\|\nu\|: \hat{\nu}=\hat{\omega} \text { on } E, \nu \in M(\mathrm{~T})\} ;
$$

here $\|\cdot\|$ is the usual total variation norm for $M(\mathrm{~T})$. The following lemma provides an effective means of computing quotient norms.

LEMMA A. For any $\omega \in M(\mathrm{~T})$ and $E \subset \mathbf{Z}$

$$
\|\omega\|_{E}=\sup \left\{\left|\sum_{-m}^{m} c_{n} \hat{\omega}(n)\right|:\left\|\sum_{-m}^{m} c_{n} e^{-i n \theta}\right\|_{\infty} \leq 1, n \in E \text { for all } n\right\} .
$$

Proof. Fix $\omega \in M(\mathbf{T})$ and $E \subset \mathbf{Z}$; that

$$
\sup \left\{\left|\sum_{-m}^{m} c_{n} \hat{\omega}(n)\right|:\left\|\sum_{-m}^{m} c_{n} e^{-i n \theta}\right\|_{\infty} \leq 1, n \in E \text { for all } n\right\} \leq\|\omega\|_{E}
$$

is obvious; to confirm the reverse inequality simply apply the Hahn-Banach and Riesz representation theorems.

The next lemma describes the behaviour at infinity of the quotient norms of a measure.

Received by the editors July 13, 1981.

1980 Mathematics Subject Classification. Primary 42A05. 
LEMMA L. For any natural number $n$ put $A_{n}=[n, \infty) \cup(-\infty,-n] \subset \mathbf{Z}$; if $\omega \in$ $M(\mathbf{T})$ then $\lim _{n \rightarrow \infty}\|\omega\|_{A_{n}}=\left\|\omega_{s}\right\|$.

ProOF. Lemma $L$ is an immediate consequence of Lemma $A$ and the work of R. Doss [3].

We say $\left\langle D_{n}\right\rangle_{1}^{\infty}$ is a sequence of positive dyadic intervals in $\mathbf{Z}$ if there exists a sequence $\left\langle n_{k}\right\rangle_{1}^{\infty} \subset \mathbf{Z}^{+}, n_{k+1} / n_{k} \geq 2$ for all $k$ and $D_{k}=\left[n_{2 k}, n_{2 k+1}\right)=\left\{m \in \mathbf{Z}: n_{2 k} \leq\right.$ $\left.m<n_{2 k+1}\right\}$. We say $\left\langle D_{n}\right\rangle_{1}^{\infty}$ is a sequence of symmetric dyadic intervals in $\mathbf{Z}$ if there exists a sequence $\left\langle n_{k}\right\rangle_{1}^{\infty} \subset \mathbf{Z}^{+}, n_{k+1} / n_{k} \geq 2$ for all $k$ and $D_{k}=\left[n_{2 k}, n_{2 k+1}\right) \cup$ $\left(-n_{2 k+1},-n_{2 k}\right] \subset \mathbf{Z}$.

Our quantitative generalization of the F. and M. Riesz Theorem is then

THEOREM V. There is a constant $C>0$ such that for any sequence $\left\langle D_{n}\right\rangle_{1}^{\infty}$ of symmetric dyadic intervals in $\mathbf{Z}$ and any $\mu \in H^{1}(\mathbf{T})$

$$
\sum_{k=1}^{\infty} \frac{\|\mu\|_{D_{k}}}{k} \leq C\|\mu\| \text {. }
$$

The statement of Theorem $\mathrm{V}$ occurred to the authors after a reading of [4]; notice that Theorem $R$ is an immediate consequence of Theorem $V$ and Lemma L. For results related to Theorem $\mathrm{V}$ see comment (e) at the end of this paper. The proof of Theorem $\mathrm{V}$ depends upon this result:

LEMMA N (cf. $[1,2,6])$. Let $\left\langle a_{n}\right\rangle_{1}^{Q}$ be a sequence of complex numbers and $\left\langle t_{n}\right\rangle_{1}^{Q}$ a sequence of trigonometric polynomials on $\mathbf{T}$ such that $\sum_{1}^{Q}\left|a_{n}\right| \leq 1,\left\|t_{n}\right\|_{\infty} \leq 1$ for $n=1,2, \ldots, Q$, and $\operatorname{supp} \hat{t}_{i} \cap \operatorname{supp} \hat{t}_{j}=\varnothing$ for $i \neq j$. Put $\beta=\sum_{1}^{Q}\left|a_{n}\right|^{2}$. Then given $\epsilon>0$, there exists a polynomial $p(z)=b_{1} z+\cdots+b_{m} z^{m}$ such that

$$
\frac{1}{5}\left|\sum_{1}^{Q} a_{n} t_{n}\right|+\left|\exp \left(-\frac{\sqrt{2}}{4} \beta^{1 / 2}\right)+p\left(\left\{\sum_{i \neq j}\left(a_{i} t_{i}\right)\left(\overline{a_{j} t_{j}}\right)\right\}^{+}\right)\right|<1+\epsilon ;
$$

here $\left(\sum_{i \neq j}\left(a_{i} t_{i}\right)\left(\overline{a_{j} t_{j}}\right)\right)^{+}$is the analytic projection of $\sum_{i \neq j}\left(a_{i} t_{i}\right)\left(\overline{a_{j} t_{j}}\right)$.

Proof of LemMA N. Put $f=\sum_{1}^{Q} a_{i} t_{i}$; then

$$
|f|^{2}=\sum\left|a_{i}\right|^{2}\left|t_{i}\right|^{2}+2 \operatorname{Re}\left\{\left(\sum_{i \neq j} a_{i} t_{i} \overline{a_{j} t_{j}}\right)^{+}\right\} .
$$

Given $\epsilon>0$ choose $\delta>0$ so that

$$
\left|\exp \left(-\frac{\sqrt{2}}{4}(\beta+\delta)^{1 / 2}\right)-\exp \left(-\frac{\sqrt{2}}{4} \beta^{1 / 2}\right)\right|<\frac{\epsilon}{2}
$$

and define $h=\beta+\delta+2\left(\sum_{i \neq j} a_{i} t_{i} \overline{a_{j} t_{j}}\right)^{+}$; notice that $\operatorname{Re} h \geq|f|^{2}+\delta$ because $\left\|t_{i}\right\|_{\infty} \leq$ $1(i=1,2, \ldots, Q)$. We gather from this that there exists a disc $D_{R} \subset \mathbf{C}$ centered about $R>0$ which lies entirely in the open right half plane such that the range of $h$ is contained in $D_{R}$ and $\beta+\delta \in D_{R}$.

Put $H(z)=z^{1 / 2}$; then $\exp (-\sqrt{2} H(z) / 4)$ is analytic on $D_{R}$ and so it follows that there is a polynomial $F(z)=c_{0}+c_{1}(z-R)+\cdots+c_{m}(z-R)^{m}$ such that for all 
$z \in D_{R}$

$$
\left|\exp \left(-\frac{\sqrt{2}}{4} H(z)\right)-F(z)\right|<\frac{\epsilon}{4} .
$$

Since $\beta+\delta \in D_{R}$ and Range $h \subset D_{R}$ we infer via inequalities (1.1) and (1.2) that

$$
\left|\exp \left(-\frac{\sqrt{2}}{4} H(h)\right)-\exp \left(-\frac{\sqrt{2}}{4} \beta^{1 / 2}\right)-p\left(\sum_{i \neq j}\left(a_{i} t_{i}\right)\left(a_{j} t_{j}\right)^{+}\right)\right|<\epsilon
$$

for some polynomial of the form $p(z)=b_{1} z+b_{2} z^{2}+\cdots+b_{m} z^{m}$.

To complete the proof of our lemma observe that

$$
\begin{aligned}
\left|\exp \left(-\frac{\sqrt{2}}{4} H(h)\right)\right|+\frac{1}{5}\left|\sum_{1}^{Q} a_{i} t_{i}\right| & \leq \exp \left(-\frac{\sqrt{2}}{4} \operatorname{Re} H(h)\right)+\frac{1}{5}|f| \\
& \leq \exp \left(-\frac{|f|}{4}\right)+\frac{1}{5}|f|
\end{aligned}
$$

because $\operatorname{Re} H(h) \geq|f| / \sqrt{2}$. Inasmuch as $\exp (-x / 4)+x / 5 \leq 1$ for $0 \leq x \leq 1$, we have confirmed

$$
\left|\exp \left(-\frac{\sqrt{2}}{4} H(h)\right)\right|+\frac{1}{5}\left|\sum_{1}^{Q} a_{i} t_{i}\right| \leq 1 .
$$

The conclusion of our lemma now follows from inequalities (1.3) and (1.4).

We now turn to the proof of Theorem V: Let $\left\langle D_{k}\right\rangle_{1}^{\infty}$ be any sequence of symmetric dyadic intervals in $\mathrm{Z}$ and let $\mu \in H^{1}(\mathbf{T})$. Put $a_{k}=1 / k(k=1,2, \ldots)$; by breaking the sequence $\left\langle a_{k}\right\rangle_{1}^{\infty}$ into the blocks $\left\{a_{1}\right\},\left\{a_{2}, a_{3}\right\},\left\{a_{4}, a_{5}, a_{6}, a_{7}\right\}, \ldots$, we may choose $\left\{Q_{1}<Q_{2}<\cdots\right\} \subset \mathbf{Z}^{+}$as follows:

$$
\left\{a_{1}^{2}+a_{2}^{2}+\cdots+a_{Q_{1}}^{2}\right\}^{1 / 2}+\left\{a_{Q_{1}+1}^{2}+\cdots+a_{Q_{2}}^{2}\right\}^{1 / 2}+\cdots \leq \frac{1}{1-(\sqrt{2})^{-1}}
$$

and

$$
a_{1}+a_{2}+\cdots+a_{Q_{1}} \leq 1, \quad a_{Q_{1}+1}+\cdots+a_{Q_{2}} \leq 1 \text {, etc. }
$$

Let $t_{k}$ satisfy supp $\hat{t}_{k} \subset D_{k},\left\|t_{k}\right\|_{\infty} \leq 1$ with $\int_{\mathrm{T}} t_{k}(\theta) d \mu(\theta) \equiv \hat{\mu}\left(t_{k}\right)=\|\mu\|_{D_{k}}$. Given $\epsilon>0,\left\langle a_{k}\right\rangle_{Q_{j+1}}^{Q_{j+1}}$ and $\left\langle t_{k}\right\rangle_{Q_{j}+1}^{Q_{j+1}}$, define $\beta_{j}=\sum_{Q_{j+1}}^{Q_{j+1}} a_{k}^{2}(j=1,2, \ldots)$ and put $\alpha_{j}=$ $\exp \left(-\sqrt{2} \beta_{j}^{1 / 2} / 4\right)$; then (using (2)) select polynomials of the form $p_{j}=b_{j 1} z+\cdots+$ $b_{j m} z^{m}$ such that

$$
\frac{1}{5}\left|\sum_{Q_{j}+1}^{Q_{j+1}} a_{k} t_{k}\right|+\left|\alpha_{j}+p_{j}\left(\left\{\sum_{Q_{j}+1 \leq i \neq j \leq Q_{j+1}}\left(a_{i} t_{i}\right)\left(\overline{a_{j} t_{j}}\right)\right\}^{+}\right)\right| \leq 1+\epsilon^{2^{j-1}} .
$$

Such a selection of polynomials $p_{j}$ is always possible by Lemma $\mathrm{N}$.

Put $F_{0}=\frac{1}{5} \sum_{k=1}^{Q_{1}} a_{k} t_{k}$ and for $n=1,2, \ldots$ define

$$
F_{n}=\frac{1}{5} \sum_{Q_{n}+1}^{Q_{n+1}} a_{k} t_{k}+F_{n-1}\left\{\alpha_{n}+p_{n}\left(\left\{\sum_{Q_{n}+1 \leq i \neq j \leq Q_{n+1}}\left(a_{i} t_{i}\right)\left(\overline{a_{j} t_{j}}\right)\right\}^{+}\right)\right\}
$$


then it is not hard to see that condition (3) implies

$$
\left\|F_{n}\right\|_{\infty} \leq \frac{1}{1-\epsilon} \quad(n=1,2, \ldots) .
$$

So, on the one hand,

$$
\left|\int_{\mathbf{T}} F_{n}(\theta) d \mu(\theta)\right| \leq \frac{1}{1-\epsilon}\|\mu\|
$$

while on the other,

(5) $5 \int_{\mathbf{T}} F_{n}(\theta) d \mu(\theta)=\sum_{Q_{n}+1}^{Q_{n+1}} a_{k} \hat{\mu}\left(t_{k}\right)+\alpha_{n} \sum_{Q_{n-1}+1}^{Q_{n}} a_{k} \hat{\mu}\left(t_{k}\right)$

$$
+\alpha_{n} \alpha_{n-1} \sum_{Q_{n-2}+1}^{Q_{n-1}} a_{k} \hat{\mu}\left(t_{k}\right)+\cdots+\left(\alpha_{n} \alpha_{n-1} \cdots \alpha_{1}\right) \sum_{1}^{Q_{1}} a_{k} \hat{\mu}\left(t_{k}\right),
$$

because $\mu \in H^{1}(\mathbf{T})$ and the sequence $\left\langle D_{k}\right\rangle_{1}^{\infty}$ is dyadic. As a consequence of (4) and (5), we obtain

$$
\frac{5}{1-\epsilon}\|\mu\| \geq\left(\alpha_{n} \alpha_{n-1} \cdots \alpha_{1}\right)\left\{\sum_{Q_{n}+1}^{Q_{n+1}} a_{k} \hat{\mu}\left(t_{k}\right)+\cdots+\sum_{1}^{Q_{1}} a_{k} \hat{\mu}\left(t_{k}\right)\right\} .
$$

It now follows from inequalities (1) and (6) that

$$
5 \exp (2 \sqrt{2}-2)^{-1} \cdot\|\mu\| \geq(1-\epsilon) \sum_{1}^{Q_{n+1}} a_{k} \hat{\mu}\left(t_{k}\right)
$$

consequently $17\|\mu\| /(1-\epsilon) \geq \sum_{1}^{Q_{n+1}} \frac{1}{k} \hat{\mu}\left(t_{k}\right)$, and this, in turn, implies that

$$
\sum_{1}^{\infty} \frac{1}{k}\|\mu\|_{D_{k}} \leq 17\|\mu\| \text {. }
$$

COMMENTS. (a) As the proof of Theorem V shows, we can relax the gap condition on the intervals somewhat, and also change the sequence $\langle 1 / k\rangle_{1}^{\infty}$ to any sequence $\left\langle a_{k}\right\rangle_{1}^{\infty} \subset \mathbf{Z}^{+}$satisfying conditions analogous to (1) and (2).

(b) The obvious analogue of Theorem $\mathrm{V}$ remains valid for the additive group of real numbers $\mathbf{R}$.

(c) Let $G$ be a compact abelian group with character group $\Gamma$; suppose $\phi: \Gamma \rightarrow \mathbf{R}$ is a nontrivial homomorphism. We say $\left\langle_{\phi} D_{k}\right\rangle_{1}^{\infty}$ is a sequence of symmetric $\phi$-dyadic intervals in $\Gamma$ if there exists a sequence $\left\langle D_{k}\right\rangle_{1}^{\infty}$ of symmetric dyadic intervals in $\mathbf{Z}$ such that for each $k_{\phi} D_{k}=\phi^{-1}\left(D_{k}\right)$. Then, there is a constant $C>0$ such that for any sequence $\left\langle_{\phi} D_{k}\right\rangle_{1}^{\infty}$ of symmetric $\phi$-dyadic intervals in $\Gamma$ and any $\mu \in H_{\phi}^{1}(G)=$ $\{\nu \in M(G): \hat{\nu}(\gamma)=0$ for all $\gamma \in \Gamma$ such that $\phi(\gamma)<0\}$

$$
\sum_{k=1}^{\infty} \frac{\|\mu\|_{\phi} D_{k}}{k} \leq C\|\mu\| \text {. }
$$

An immediate consequence of the above inequality is that if $\mu \in H_{\phi}^{1}(G)$ then $\mu$ translates continuously in the direction of $\phi$ and this in turn implies that $\mu_{s} \in$ $H_{\phi}^{1}(G)$; see [6] for definitions and appropriate references. 
(d) We say $\left\langle D_{k}\right\rangle_{1}^{\infty}$ is a sequence of symmetric dyadic boxes in $\mathbf{Z}^{n}$ if there exists a sequence $\left\langle m_{k}\right\rangle_{1}^{\infty} \subset \mathbf{Z}^{+}, m_{k+1} / m_{k} \geq 2$ for all $k$ and

$$
D_{k}=\left\{\left(y_{1}, \ldots, y_{n}\right):\left|y_{i}\right| \in\left[m_{2 k}, m_{2 k+1}\right) \cup\{0\}\right\} \backslash\{0\} \subset \mathbf{Z}^{n} .
$$

Then, there is a constant $C>0$ such that for any sequence $\left\langle D_{k}\right\rangle_{1}^{\infty}$ of symmetric dyadic boxes in $\mathbf{Z}^{n}$ and any $\mu \in M\left(\mathbf{T}^{n}\right)$

$$
\sum_{1}^{\infty} \frac{\|\mu\|_{D_{k}}}{k} \leq C\|\mu\|
$$

provided supp $\hat{\mu} \subset\left\{\left(x_{1}, \ldots, x_{n}\right): x_{i} \in \mathbf{Z}, x_{i} \geq 0\right.$ for all $\left.i\right\}$. It is not hard to see that the above inequality is a quantitative generalization of S. Bochner's several-variable extension of the F. and M. Riesz Theorem.

To obtain the proofs of (c) and (d) it suffices to repeat the proof of Theorem $\mathrm{V}$ after making appropriate modifications in Lemmas $\mathrm{A}, \mathrm{L}$, and $\mathrm{N}$.

(e) A sequence of symmetric dyadic intervals $\left\langle D_{k_{1}}\right\rangle^{\infty}, D_{k}=\left[n_{2 k}, n_{2 k+1}\right) \cup$ $\left(-n_{2 k+1},-n_{2 k}\right]$, is said to be standard if there exists a $\lambda>0$ such that $n_{k+1} / n_{k}<$ $\lambda$ for all $k$. Notice that Theorem $\mathrm{V}$ for standard dyadic intervals is a special case of known Littlewood-Paley type inequalities [8]; however, in order to obtain our applications it is important that we not restrict ourselves to standard dyadic intervals.

The original proof of Theorem V used a linear fractional transformation rather than the exponential function of the present argument; the use of the exponential function makes more explicit the connection between the method of proof of Theorem $\mathrm{V}$ and [5].

\section{REFERENCES}

1. P. Cohen, On a conjecture of Littlewood and idempotent measures, Amer. J. Math. 82 (1960), 191-192.

2. H. Davenport, On a theorem of $P$. J. Cohen, Mathematika 7 (1960), 93-97.

3. R. Doss, On the transform of a singular or absolutely continuous measure, Proc. Amer. Math. Soc. 19 (1968), 361-363.

4. J. J. F. Fournier, On a theorem of Paley and the Littlewood conjecture, Ark. Mat. 17 (1979), 199-216.

5. O. C. McGehee, L. Pigno and B. Smith, Hardy's inequality and the $L^{1}$ norm of exponential sums, Ann. of Math. (2) 113 (1981), 613-618.

6. L. Pigno and B. Smith, On measures of analytic type, Proc. Amer. Math. Soc. 82 (1981), 541-547.

7. F. and M. Riesz, Über die Randwerte einer analytischen Funktion, Quatrième Congrès des Math. Scand. Stockholm (1916), 27-44.

8. E. M. Stein, Classes $H^{p}$ multiplicateurs et fonctions de Littlewood-Paley, C. R. Acad. Sci. Paris Sér. A 263 (1966), 780-781.

DEPARTMENT OF MATHEMATICS, KANSAs STATE UNIVERSITY, MANHATTAN, KANSAS 66506 (Current address of Louis Pigno) 61761

Department of Mathematics, Illinois State University, Normal, Illinois

Current address (Brent Smith): Department of Mathematics, California Institute of Technology, Pasadena, California 91125 